\title{
Electrical properties of thermally evaporated tellurium thin films
}

\author{
T BALASUBRAMANIAM ${ }^{\dagger}$, SA K NARAYANDASS* and \\ D MANGALARAJ \\ Department of Physics, Bharathiar University, Coimbatore 641046 , India \\ ${ }^{\dagger}$ Department of Physics, Kongunadu Arts and Science College, Coimbatore 641 029, India \\ MS received 20 June 1996; revised 22 November 1996
}

\begin{abstract}
Resulis of dielectric and conduction properties of vacuum evaporated tellurium (Te) thin film capacitors ( $\mathrm{Al}-\mathrm{Te}-\mathrm{Al}$ ) have been reported in the frequency range $1-100 \mathrm{kHz}$ at various temperatures $(303-423 \mathrm{~K})$. Loss factor $(\tan \delta$ ) which shows a maximum with frequency increases with rise of temperature and $\tan \delta_{\text {max }}$ shift towards high frequency region. The large values of capacitance and dielectric constant $\left(\varepsilon^{\prime}\right)$ in the low frequency region indicate the possibility of an interfacial polarization mechanism. $I-V$ characteristics show ohmic, space charge limited (SCLC) and thermionic emission conduction mechanisms to operate at low, intermediate and high voltages respectively. Various transport parameters have been calculated. It has been observed that the Schottky type of conduction is predominant in the high field region and the Schottky barrier height has been determined. The Hall coefficient, Hall mobility and carrier concentration are also discussed.
\end{abstract}

Keywords. Vacuum evaporation; tellurium; dielectric; electrical properties.

\section{Introduction}

Polycrystalline thin films of tellurium find applications as IR detectors, strain sensitive devices and thin film transistors (Gutierrez et al 1962). The structure and transport properties of Te films prepared by evaporation have been widely investigated (Chaudhury and Duttaray 1965; Capers and White 1971, 1973; Chaudhury and Bose 1977; Dinno et al 1974; Kobory and Janda 1977; Okuyama et al 1977; Orlowski et al 1977; Phahle 1977). This paper deals with the results of dielectric and conduction properties of vacuum evaporated Te thin films.

\section{Experimental}

\subsection{Film preparation}

Using a coating unit (Hind Hivac 12A4), aluminium (99.99\% Balzers) was evaporated from a tungsten filament onto well cleaned glass substrates through suitable masks to form the base electrode (For cleaning the glass substrates were treated with detergents, ultrasonic agitation and isopropyl alcohol). Tellurium (99.99\% Balzers) was then evaporated from a tungsten boat to form the dielectric layer. An aluminium counter electrode was evaporated onto the dielectric through suitable masks to complete the aluminium-tellurium-aluminium structure. A working pressure of $2.7 \mathrm{mPa}$ was maintained throughout the evaporation processes.

*Author for correspondence 


\subsection{Measurements}

The thicknesses of the dielectric films were measured with a multiple beam interferometer (Tolansky 1948). Structural studies were conducted using a Seifert X-ray diffractometer with filtered $\mathrm{CuK}_{\alpha}$ radiation $(\lambda=0 \cdot 15406 \mathrm{~nm})$. Measurements of capacitance $\left(C_{\mathrm{p}}\right)$ and dissipation factor $(\tan \delta)$ in the frequency range $1-10 \mathrm{kHz}$ were carried out using a Radart $0.1 \%$ universal bridge coupled with an audio oscillator and Philips PM 3206, $15 \mathrm{MHz}$ oscilloscope. Studies in the frequency range $10-100 \mathrm{kHz}$ were carried out using a multifrequency LCR meter (Hewlett-Packard 4275A). The temperature of the sample was measured with a pre-calibrated copper constantan thermocouple. The parallel equivalent conductance $\left(G_{\mathrm{p}}=W C_{\mathrm{p}} \tan \delta\right)$ was calculated at different temperatures from the measured values of capacitance $C$ and $\tan \delta$. From the calculated values of $G_{\mathrm{p}}$ the values of conductivity $(\sigma)$ were calculated knowing the thickness $(d)$ and the area $(A)$ of the capacitor. As described earlier (Nikam and Aher 1993, 1994), the current across the capacitor as a function of the applied voltage from a d.c. power supply was measured in a rotary vacuum, at different temperatures from 303 to $403 \mathrm{~K}$ using an electrometer amplifier and digital multimeter. For Hall measurements, uniform magnetic field of strength $4 \mathrm{~K}$ Gauss was used. The Hall voltage was measured using a vernier potentiometer by changing the magnetic field and sample current directions. The sample current was measured from the voltage drop across a suitable resistance. Integral method was employed for the thermo e.m.f. measurement.

\section{Results and discussion}

\subsection{Structure}

Figure 1 shows the X-ray diffractogram for two different film thicknesses. From XRD studies the structure was found to be polycrystalline in nature. The grain sizes were evaluated by knowing the full width half maximum (FWHM) of the diffraction peaks and their corresponding $2 \theta$ values and by making use of the Scherrer's formula. It was found that the value varied from $2-19 \mathrm{~nm}$ for films of thickness $88-295 \mathrm{~nm}$ respectively.

\subsection{Annealing effect}

Freshly deposited films (unannealed) have many defects and impurities. Application of a mild heat treatment would anneal out some of the defects such as voids, grain boundaries, dislocations, stresses, inhomogeneity etc. Annealing in vacuum improves the dielectric properties. Stabilization of the capacitors could be achieved by repeated annealing cycles (slow heating and cooling). Two cycles of annealing, about $343 \mathrm{~K}$ for each cycle $1 \mathrm{~h}$ were required for tellurium thin films. Films of reproducible properties is obtained if annealed at $\sim 343 \mathrm{~K}$ (Roberts et al 1974). Figure 2 shows the changes in capacitance with frequency for different annealing cycles for the tellurium film of thickness $88 \mathrm{~nm}$. The capacitance was found to decrease with repeated annealing cycles. This is because annealing is a process related with stress relief and local structural rearrangements (Nakane et al 1979) resulting in recovery of stoichiometry in the films. Cycle after cycle, the defects are reduced gradually and each atom occupies a stable position in the interior of the film until the dielectric properties attain a stable 


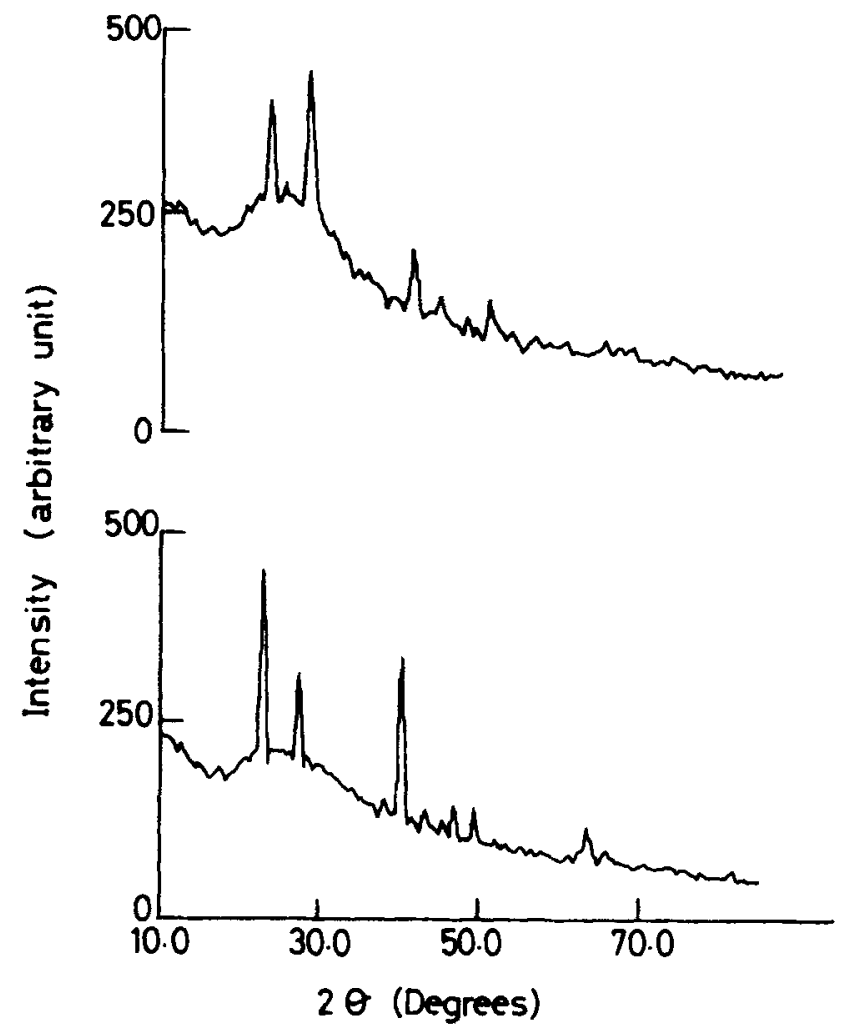

Figure 1. X-ray diffractogram.

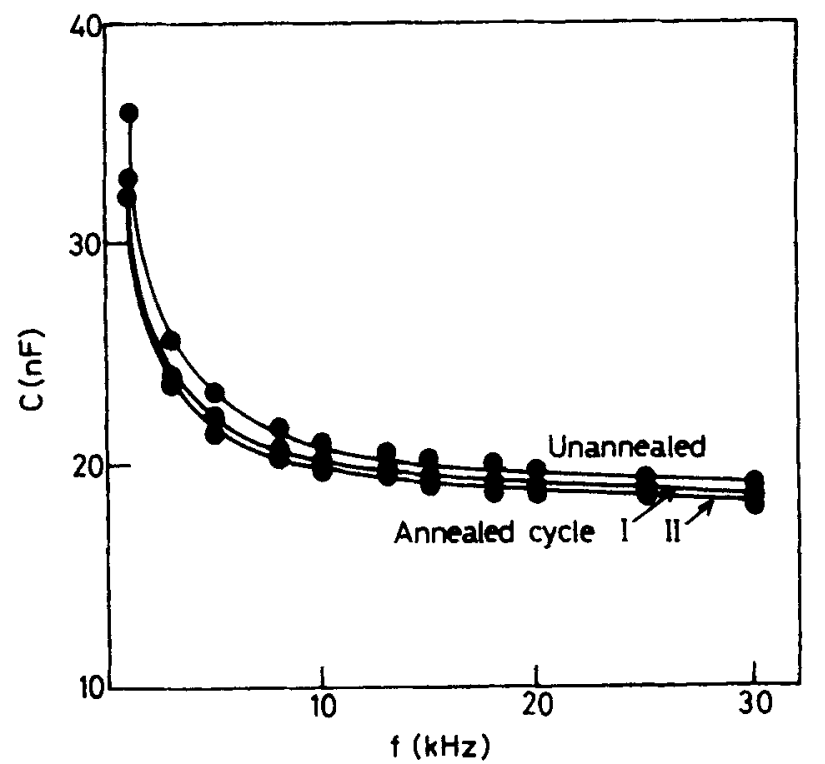

Figure 2. Variation in capacitance with frequency for different annealing cycles. 


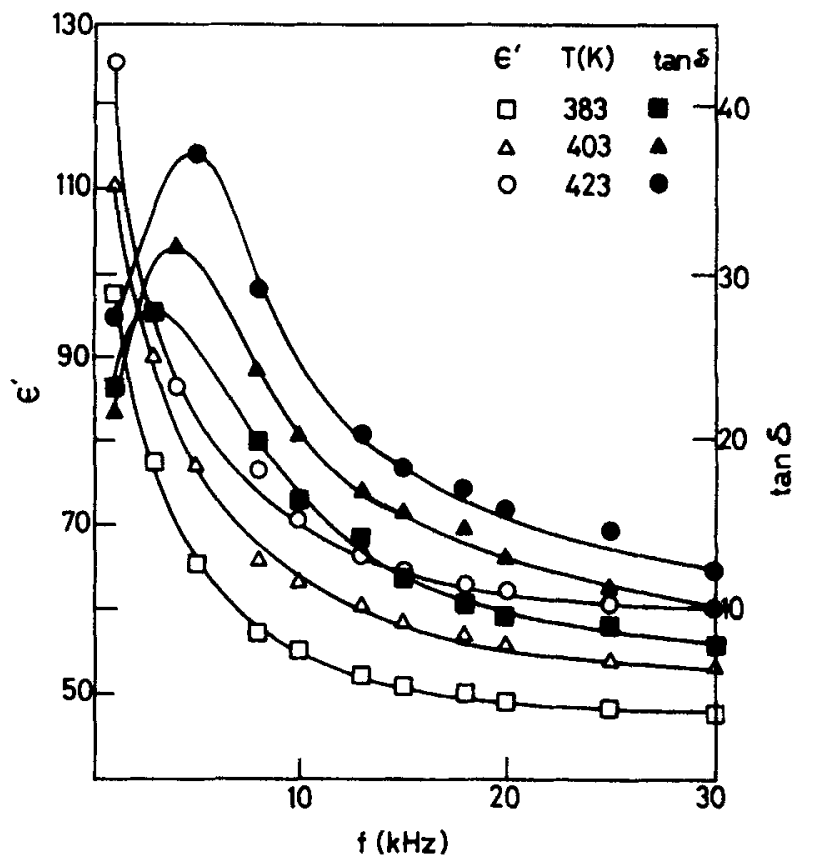

Figure 3. Variations of $\varepsilon^{\prime}$ and $\tan \delta$ with frequency at different temperatures.

value. Similar behaviour has been reported (Kannan et al 1990; Easwaran et al 1992) for $\mathrm{Nd}_{2} \mathrm{O}_{3}$ and $\mathrm{LiNbO}_{3}$ thin films.

\subsection{Dielectric properties}

The dielectric constant $\left(\varepsilon^{\prime}\right)$ was evaluated from the knowledge of capacitance $C$, film thickness $(d)$, free-space permittivity $\left(\varepsilon_{0}\right)$ and area of the capacitor $(A)$, using the relation,

$$
c d=\varepsilon^{\prime} \varepsilon_{0} A,
$$

(Peek et al 1985). The $\varepsilon^{\prime}$ value for a film of thickness $88 \mathrm{~nm}$ has been found to be 66 at $1 \mathrm{kHz}$ and at room temperature.

The variations of $\varepsilon^{\prime}$ and $\tan \delta$ with frequency at various temperatures are presented in figure 3. $\varepsilon^{\prime}$ decreases with frequency whereas $\tan \delta$ increases initially, attains a maximum and then decreases with frequency. It is interesting to note that the peak shifts towards high frequency region with the increase of temperature. This shift of the relaxation peak towards the higher frequency region with the increase of temperature is found to be in agreement with the Debye theory of dipole orientation (Rapos et al 1976).

The variations of capacitance with frequency at different temperatures are presented in figure 4. The large increase of capacitance with decrease of frequency can be explained on the basis of charge carriers being blocked at the cathodes. The space charge layer eventually leads to a substantial increase in capacitance. But at higher frequencies the capacitance is almost constant and it increases as the temperature is increased (Birey 1978; Mahalingam et al 1981).

The prominant loss peaks which appear in figure 5 manifests a dielectric relaxation effect in these films. The loss factor is seen to increase with increase of temperature and 


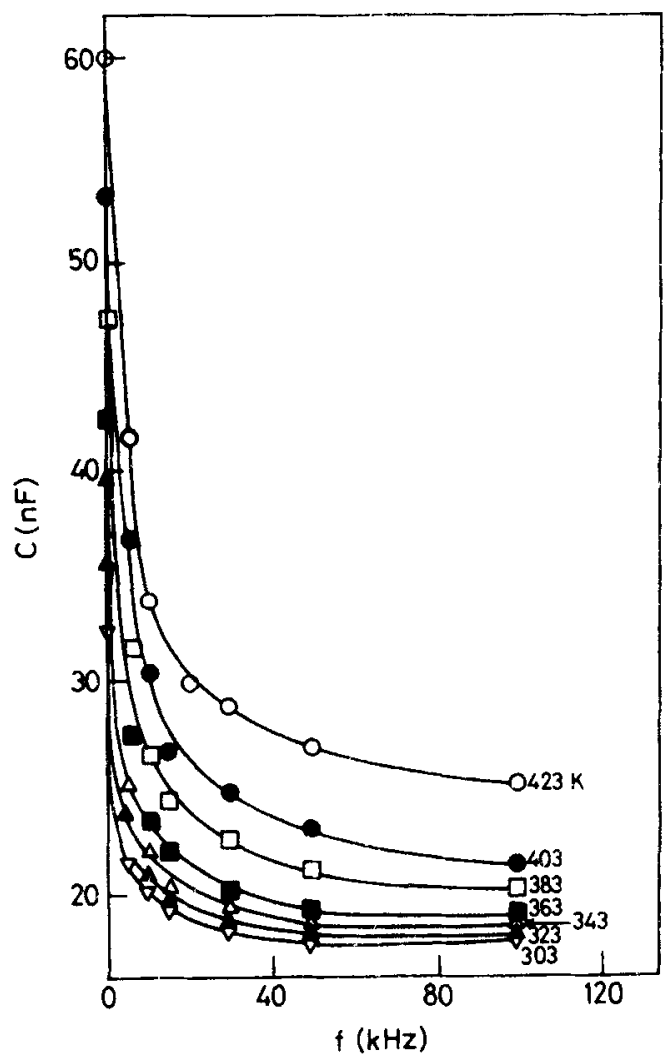

Figure 4. Variation of capacitance with frequency at different temperatures.

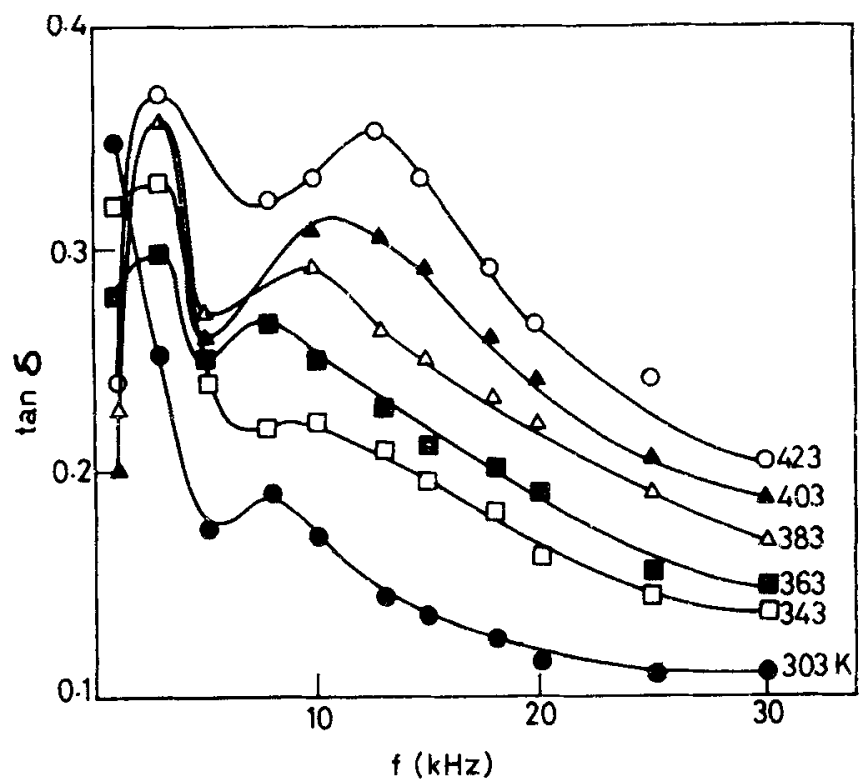

Figure 5. Variation of $\tan \delta$ against frequency. 


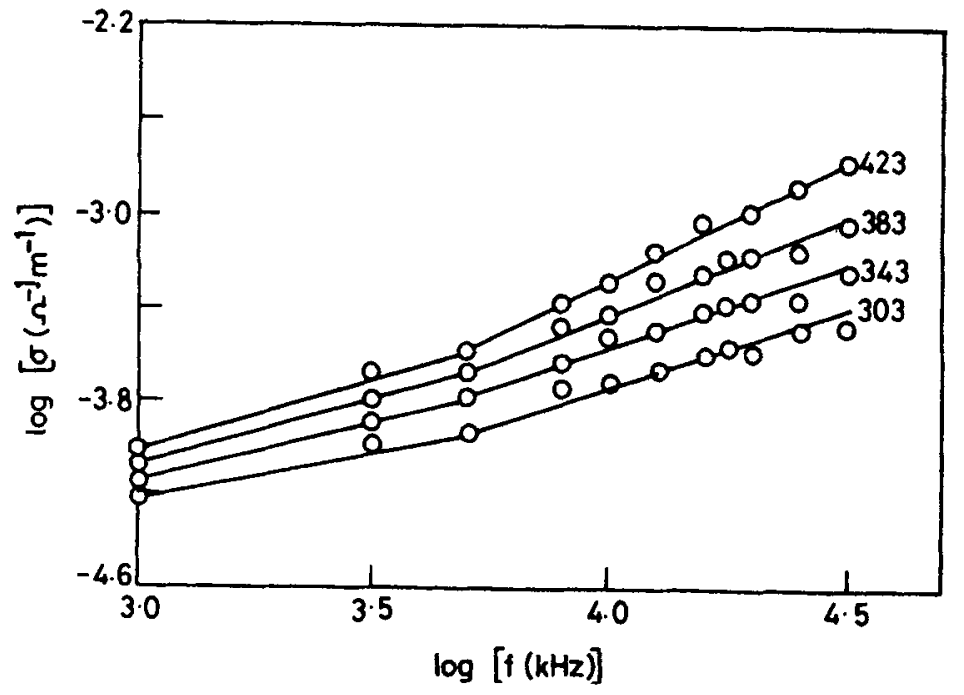

Figure 6. Plot of $\log \sigma$ vs $\log f$.

shows a maximum at each temperature which shifts toward a higher frequency as the temperature is increased (Lancaster 1972; Jonscher 1973).

\subsection{A.C. conduction}

Figure 6 shows the plot of $\log (\sigma)$ against $\log$ (freq.) at different temperatures for the same film $(d=88 \mathrm{~nm})$. The plot exhibits two regions, one below $5 \mathrm{kHz}$ and the other above $5 \mathrm{kHz}$. The a.c. conductivity varies according to the relation $G_{\mathrm{p}} \propto f^{n}$ where the value of $n$ depends on the temperature and frequency range studied. In the first region, $n$ varies from 0.4 to 0.85 . In the second region $n$ is found to vary from 1.3 to 1.9 i.e. it shows a square law dependence. The presence of two dispersive regions suggests that the mechanism responsible for a.c. conduction is of the hopping type. A similar behaviour has been observed for indium oxide films (Goswami and Goswami 1977), tellurium oxide films (Lakshminarayan et al 1982), and germanium dioxide thin films (Sasi et al 1987).

Figure 7a shows $\log (\sigma)$ vs $1 / T$ data at various frequencies. The activation energy at $10 \mathrm{kHz}$ is $0.21 \mathrm{eV}$. Figure $7 \mathrm{~b}$ shows the plot of $\log f_{\max }$ against $1 / T$, where $f_{\max }$ is the frequency at which the loss maximum is observed. The activation energy of $0.23 \mathrm{eV}$, is close to the value obtained from the data of figure $7 \mathrm{a}$.

\subsection{D.C. conduction}

Figure 8 shows the plot between $\log (I)$ and $\log (V)$ for tellurium thin film of a typical thickness $88 \mathrm{~nm}$. Three regions are observed at each temperature. In the first region (AB), at low voltages the conduction is ohmic $(I \propto V)$. In the second region $(B C)$, a trap square law $\left(I \propto V^{2}\right)$ dependence is observed. In the third region (CD), the current increases rapidly with the increase of applied voltage and the slope is seen to vary from 6 to 8 . The square law region is seen to decrease with increase in temperature. This is 


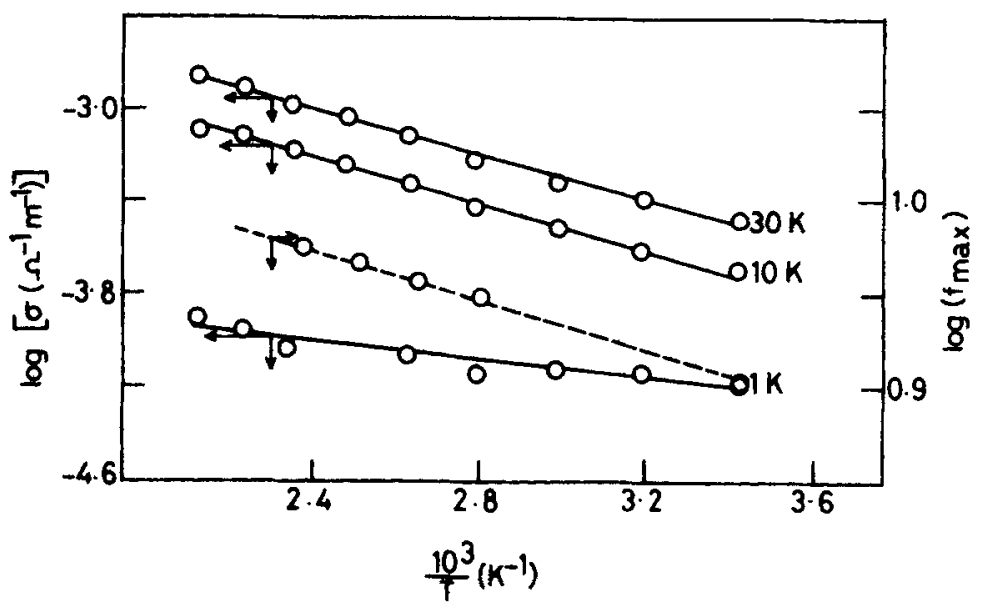

Figure 7. a. Plot of $\log \sigma$ vs $1 / T$ and b. plot of $\log f_{\max }$ vs $1 / T$.

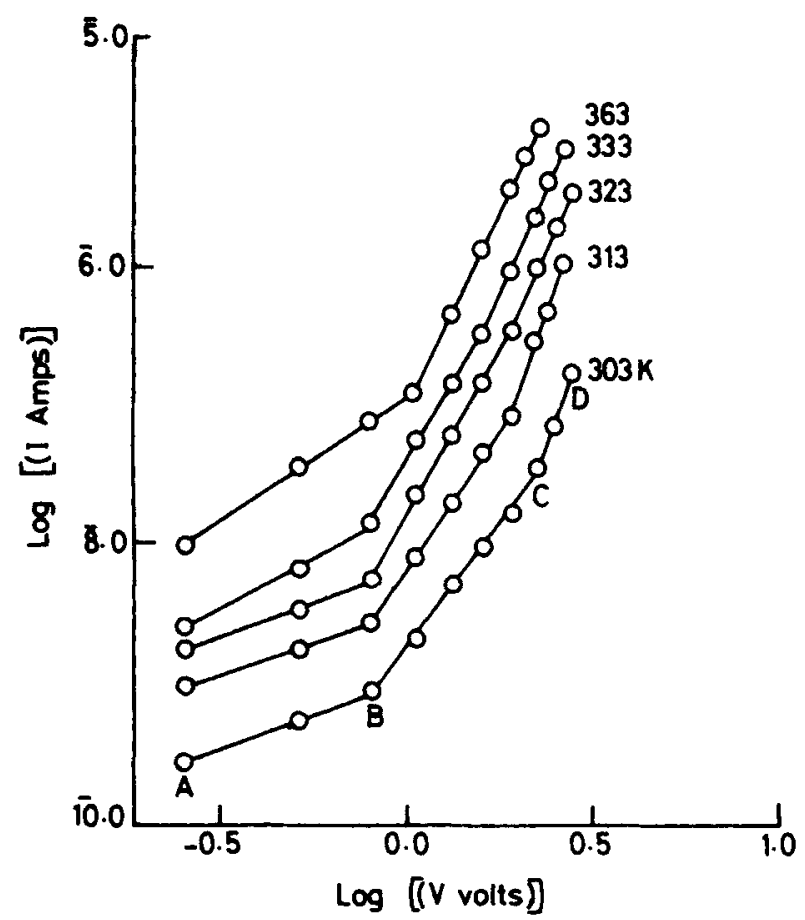

Figure 8. Variation of $\log I$ with $\log V$.

due to the predominance of injected free carriers over trapped carriers at higher temperatures.

Figure 9 shows the variation in current density with different film thickness at a temperature of $303 \mathrm{~K}$ in the square law region (at $4 \mathrm{~V}$ ). A straight line plot is obtained whose slope is -4 . The current requires a thickness dependence of the form $I \propto d^{-n}$, where $n$ is a parameter that depends on the trap distribution $(n \geqslant 3)$. The slope of the plot (figure 9 ) being greater than -3 confirms that the observed currents are actually 


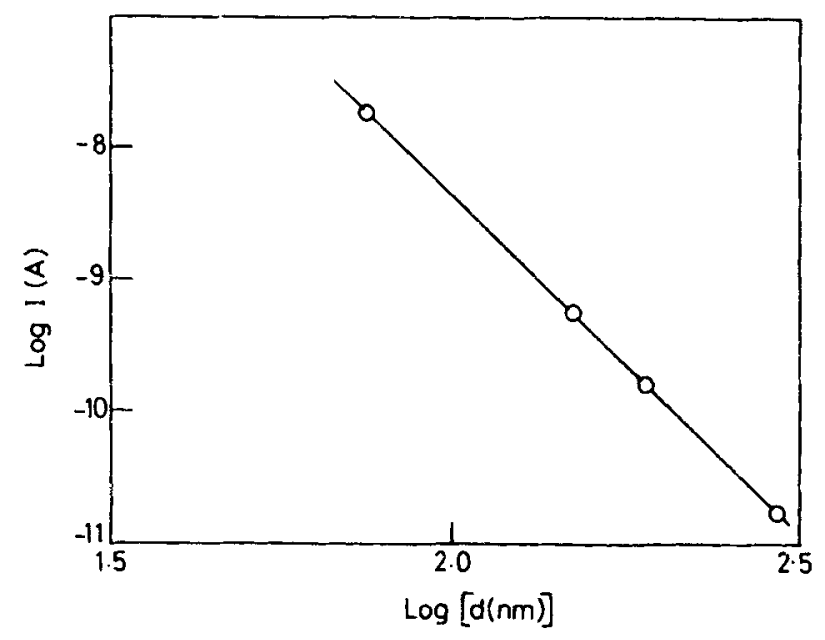

Figure 9. Variation of current density with different thicknesses.

space charge limited. The relation (Subbrayan et al 1988) for the current density in the square law region is

$$
J=(9 / 8) \mu \varepsilon^{\prime} \varepsilon_{0} \theta\left(V^{2} / d^{3}\right)
$$

where $\mu$ is the free carrier mobility, $\varepsilon_{0}$ the permittivity of free space, $\varepsilon^{\prime}$ the dielectric constant of the material of the film and

$$
\theta=N_{\mathfrak{c}} / N_{\mathrm{t}} \exp \left(-E_{\mathrm{F}} / k T\right)
$$

Here $N_{\mathrm{c}}$ is the effective density of states in the conduction band, $N_{\mathrm{t}}$ the density of shallow traps positioned at an energy $E_{\mathrm{F}}$ below the conduction band, $k$ the Boltzmann constant and $T$ the absolute temperature. Also $\theta$ is the ratio of the free electron density $n_{0}$ in the conduction band to the total electron density $\left(n_{0}+n_{1}\right)$ where $n_{1}$ is the density of the trapped electrons. Experimentally, $\theta$ is the ratio of the current density $I_{1} / A$ at the beginning of the square law region to the current density $I_{2} / A$ at the end of the square law region, where $A$ is the area of the capacitor, i.e.,

$$
I_{1} / I_{2}=\theta=n_{0} / n_{0}+n_{1}
$$

The growth of current faster than $V^{2}$ after square law region is due to the traps. Using the experimental value of $\theta$ and $\varepsilon^{\prime}=66$ in (1), the free-carrier mobility has been calculated as $0.9 \times 10^{-12} \mathrm{~m}^{2} \mathrm{~V}^{-1} \mathrm{~s}^{-1}$ at $4 \mathrm{~V}$ and at $303 \mathrm{~K}$. The equilibrium concentration of charge carriers in the conduction band is

$$
n_{0}=\left[\varepsilon^{\prime} \varepsilon_{0} \theta / q d^{2}\right] V_{\mathrm{tr}}
$$

Here $V_{\mathrm{tr}}$ is the voltage at which the transition from ohmic to the square law region takes place, $q$ the electronic charge and $d$ the thickness of the film. From (4) $n_{0}$ is calculated as $19.9 \times 10^{24} \mathrm{~m}^{-3}$. The value of $n_{1}$ from (3) has been estimated as $8.87 \times 10^{24} \mathrm{~m}^{-3}$.

Figure 10 shows the relation of $n_{0}$ and $\theta$ with inverse absolute temperature. From the slope of the straight iine graph $[\log (\theta)$ and $I / T]$, the activation energy for d.c. 


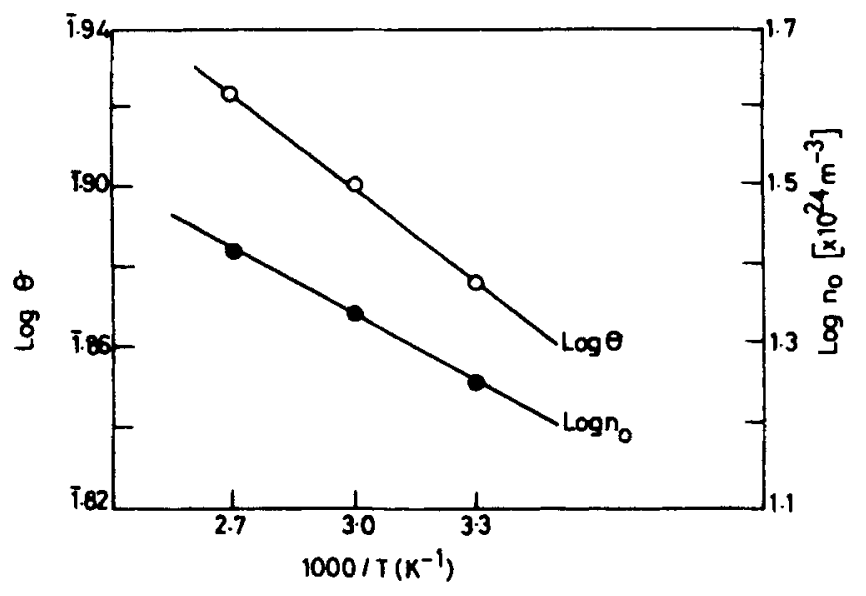

Figure 10. Variation of $n_{0}$ and $\theta$ with $1 / T$.

Table 1. $\mu, n_{0}$ and $n_{1}$ at various temperatures.

\begin{tabular}{|c|c|c|c|}
\hline $\begin{array}{l}T \\
(\mathrm{~K})\end{array}$ & $\stackrel{\mu}{\stackrel{\mu}{2}}\left(10^{-12} \mathrm{~m}^{2} \mathrm{~V}^{-1} \mathrm{~s}^{-1}\right)$ & $\begin{array}{c}n_{0} \\
\left(10^{24} \mathrm{~m}^{-3}\right)\end{array}$ & $\begin{array}{c}n_{1} \\
\left(10^{24} \mathrm{~m}^{-3}\right)\end{array}$ \\
\hline 303 & 0.91 & $19 \cdot 9$ & 8.9 \\
\hline 333 & 1.32 & $22 \cdot 1$ & 6.8 \\
\hline 363 & 1.65 & $26 \cdot 8$ & $5 \cdot 2$ \\
\hline
\end{tabular}

conduction has been calculated as $0.02 \mathrm{eV}$. From the dependence of $n_{0}$ on $1 / T$ the Fermi level $E_{\mathrm{F}}$ is determined as $0.33 \mathrm{eV}$.

Table 1 shows the above mentioned parameters as determined for a film of thickness $88 \mathrm{~nm}$ at different temperatures. Lowering of the trapping probability with a rise in temperature may be the reason for the decrease in $n_{1}$ values. The observed increase in $\mu$ with the increase of temperature is the characteristic feature of most dielectrics (Harrop 1972).

The field dependence of current density is shown in figure $11\left(\log J\right.$ vs $\left.F^{1 / 2}\right)$ Such a linear plot in the moderate high-field region indicates that the conduction mechanism may be of either Schottky or Poole-Frenkel type. The Schottky effect is the conduction of electrons by thermal excitation over the potential barrier between electrons at the Fermi level of the injecting electrode and electrons in the insulator conduction band.

$$
\left.J=J_{\mathrm{sc}} T^{2} \exp \left(\beta_{\mathrm{sc}} F^{1 / 2}-\phi\right) /(k T)\right),
$$

where $\beta_{\mathrm{sc}}$ is the Schottky field-lowering coefficient, $F$, the field and $\phi$, the barrier height.

Poole-Frenkel conduction occurs as a field emission of electrons from localized donors located at an energy, $\phi$, below the insulator conduction band. The current density in this case is

$$
J=J_{\mathrm{PF}} \exp \left(\beta_{\mathrm{PF}} F^{1 / 2}-\phi\right) /(k T) .
$$

The field-lowering coefficient, $\beta$, is given by

$$
\beta=\left[\left(e^{3} / a \pi \varepsilon_{0} \varepsilon^{\prime}\right)\right]^{1 / 2},
$$




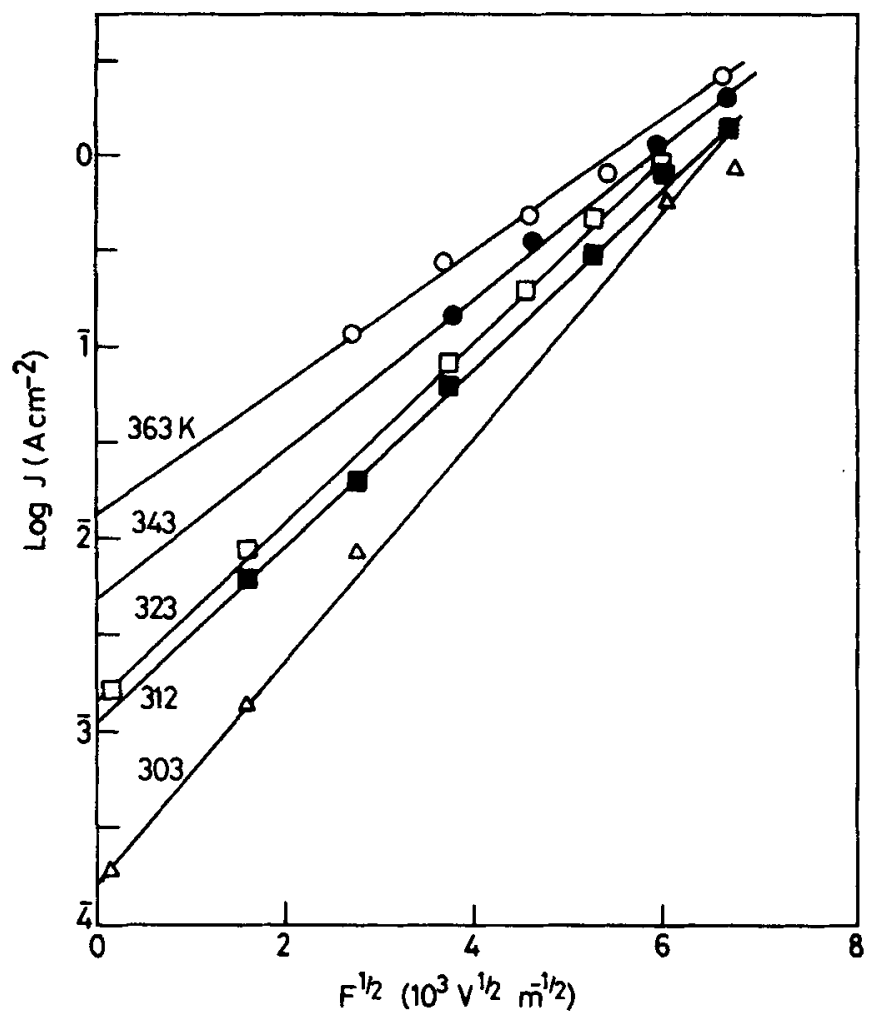

Figure 11. Plot of $\log J$ vs $F^{1 / 2}$.

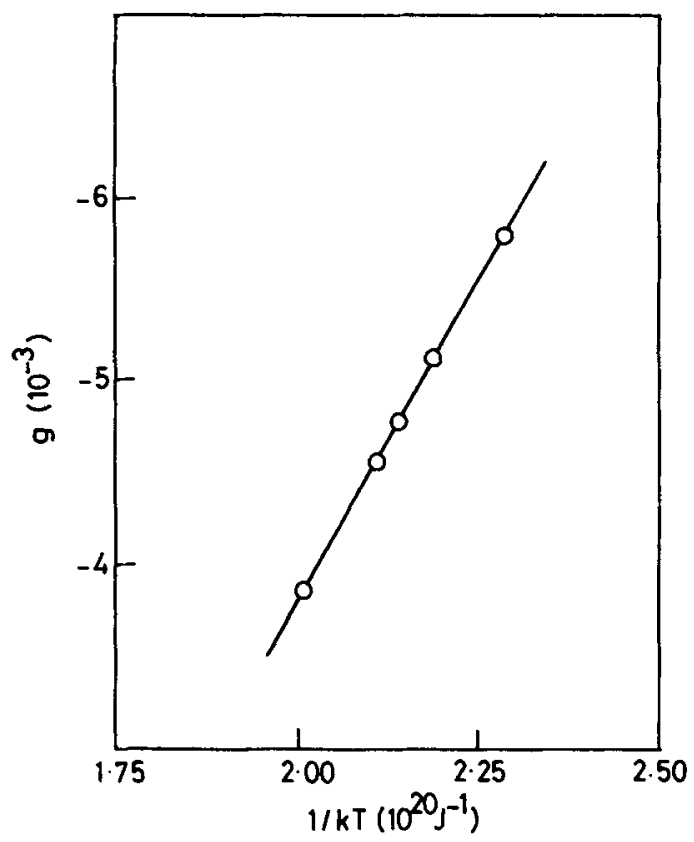

Figure 12. Plot of $g$ vs $1 / T$. 
where $e$ is the electronic charge, $a=1$ for PF emission and $a=4$ for Schottky emission, $\varepsilon_{0}$, the permittivity of free space and $\varepsilon^{\prime}$, the high frequency dielectric constant. However there could be no reason for taking the high frequency dielectric constant (Soukup and Speliotis 1970) and it has also been pointed out that $\beta_{\mathrm{sc}}$ is a function of both the low and high frequency values of dielectric constant (Hill 1971). The values thus obtained for Schottky, $\beta_{\mathrm{sc}}$, and Poole-Frenkel, $\beta_{\mathrm{PF}}$ are $0.86 \times 10^{-24}$ and $1.51 \times 10^{-24} \mathrm{eV} \mathrm{V}^{-1 / 2}$ $\mathrm{cm}^{1 / 2}$ respectively. The dielectric constant $\varepsilon^{\prime}$ for the calculation has been taken as 66 (Balasubramaniam et al 1991, 1995). The experimental value of $\beta\left(0.9 \times 10^{-24} \mathrm{eV}\right.$ $\mathrm{V}^{1 / 2} \mathrm{~cm}^{1 / 2}$ ) has been determined from the plots of $\log J$ vs $F^{1 / 2}$ (figure 11) and $g$ versus the inverse of absolute temperature (figure 12). The values of $g$ are the slopes determined from figure 11 in the high field region.

Table 2 compares the experimental and theoretical values of $\beta$. From table 2 it is clear that the experimental value is closer to the calculated $\beta_{\mathrm{sc}}$ than $\beta_{\mathrm{PF}}$. Hence, it can be proposed that the dominating conduction mechanism for thermally evaporated tellurium thin films may be of the Schottky type. However it has been reported that a mere coincidence of the experimental $\beta_{\mathrm{sc}}$ with the theoretical value cannot be taken as a deciding factor for the conduction mechanism responsible (Hughes and Jones 1974; Gould and Hogarth 1975; Kannan et al 1992). Therefore, in order to confirm the observed $\beta_{\text {sc }}$ dependence with theoretical value of $\beta$ (Reucroft and Ghosh 1974), a Schottky plot between $\log \left(J / T^{2}\right)$ and $1 / T$ (figure 13) was drawn for different voltages. The resulting straight lines observed in figure 13 confirm the Schottky type of conduction mechanism.

The zero-field current density $J_{0}$ was determined from figure 11 by extrapolating the linear plots to zero field. When $\log \left(J_{0} / T^{2}\right)$ was plotted against inverse absolute temperature (figure 14), a straight line plot was obtained and from the slope the height of the Schottky barrier was calculated as $0.06 \mathrm{eV}$. It has been shown (Gould 1982) that the slopes obtained from figure 14 for different constant voltages $(V=4 \mathrm{~V})$ is given by

$$
\text { slope }=T_{\mathrm{t}} \log \left(\varepsilon^{\prime} V / e d^{2} N_{\mathrm{t}}\right) .
$$

From the above the trap density $N_{\mathrm{t}}$ is found to be of the order of $10^{18} \mathrm{~m}^{-3}$.

\subsection{Hall effect and thermoelectric studies}

The variation of Hall coefficient $R_{\mathrm{H}}$ of the tellurium films of different thicknesses with temperature is shown in figure $15 \mathrm{a}$. It has been observed that with the increase in temperature, $R_{\mathrm{H}}$ remains more or less constant with slight variations, up to $340 \mathrm{~K}$. Thereafter a sharp fall in $R_{\mathrm{H}}$ has been observed with further increase in temperature. A confirmation of the results were obtained by earlier workers (Goswami and Ojha 1973).

Table 2. Theoretical and experimental values of $\beta$.

\begin{tabular}{lcc}
\hline & \multicolumn{2}{c}{$\beta$} \\
& $\left(10^{-24} \mathrm{eV} \mathrm{V}^{-1 / 2} \mathrm{~cm}^{1 / 2}\right)$ & \\
\hline Theoretical & Experimental \\
\hline$\beta_{\mathrm{sc}} 0.86$ & 0.90 \\
$\beta_{\mathrm{PF}} 1.51$ & \\
\hline
\end{tabular}




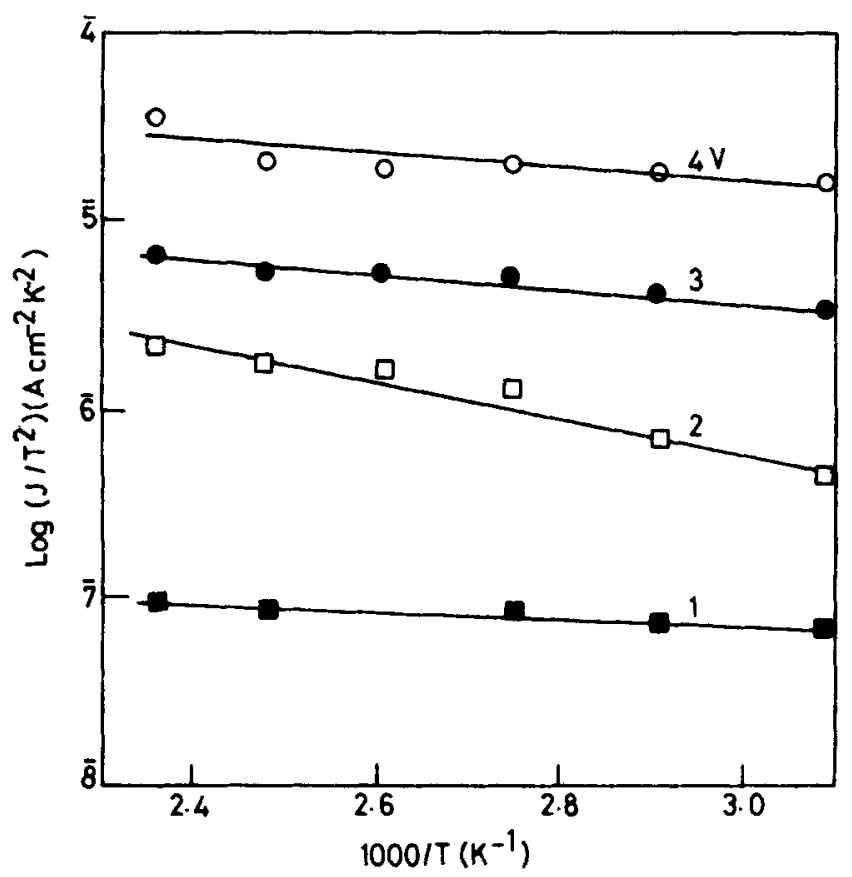

Figure 13. Plot of $\log J / T^{2}$ vs $1 / T$.

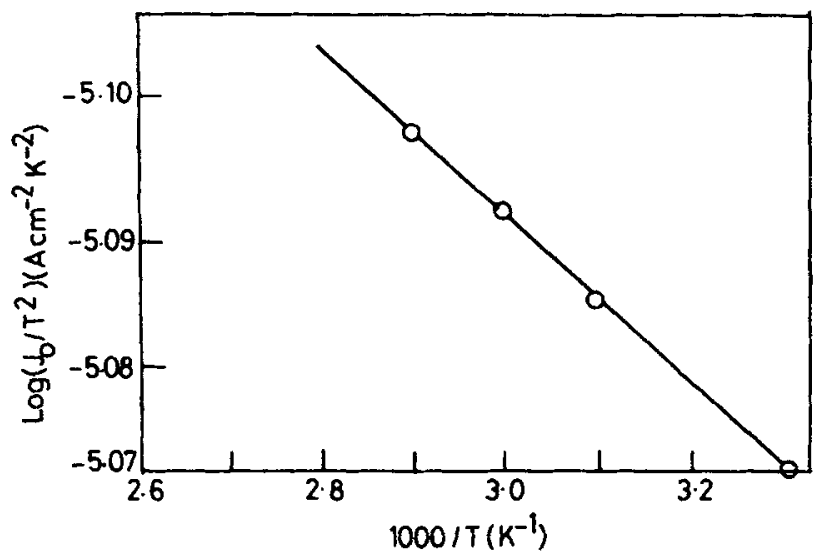

Figure 14. Plot of $\log J_{0} / T^{2}$ vs $1 / T$.

The mobility of carriers in a semiconductor depends on the scattering mechanisms. The temperature dependence of carrier mobility in tellurium films is shown in figure $15 \mathrm{~b}$. The trend of the mobility curves is more or less similar to that of the Hall coefficient i.e. remains constant up to $340 \mathrm{~K}$ and then a steep fall. Hall mobility does not appear to depend linearly on $T^{-3 / 2}$ (Leistiko et al 1965) but the trend indicates that the different types of scattering centres are active in different temperature ranges. The mobility is governed by intercrystalline grain boundaries (Capers and White 1973).

Also it has been observed that the carrier mobility increases slightly with increase in film thickness. Similar observations have been made for indium antimonide thin films (Wieder 1965) and lead tellurium thin films (Berry and Jayadereiah 1969). 


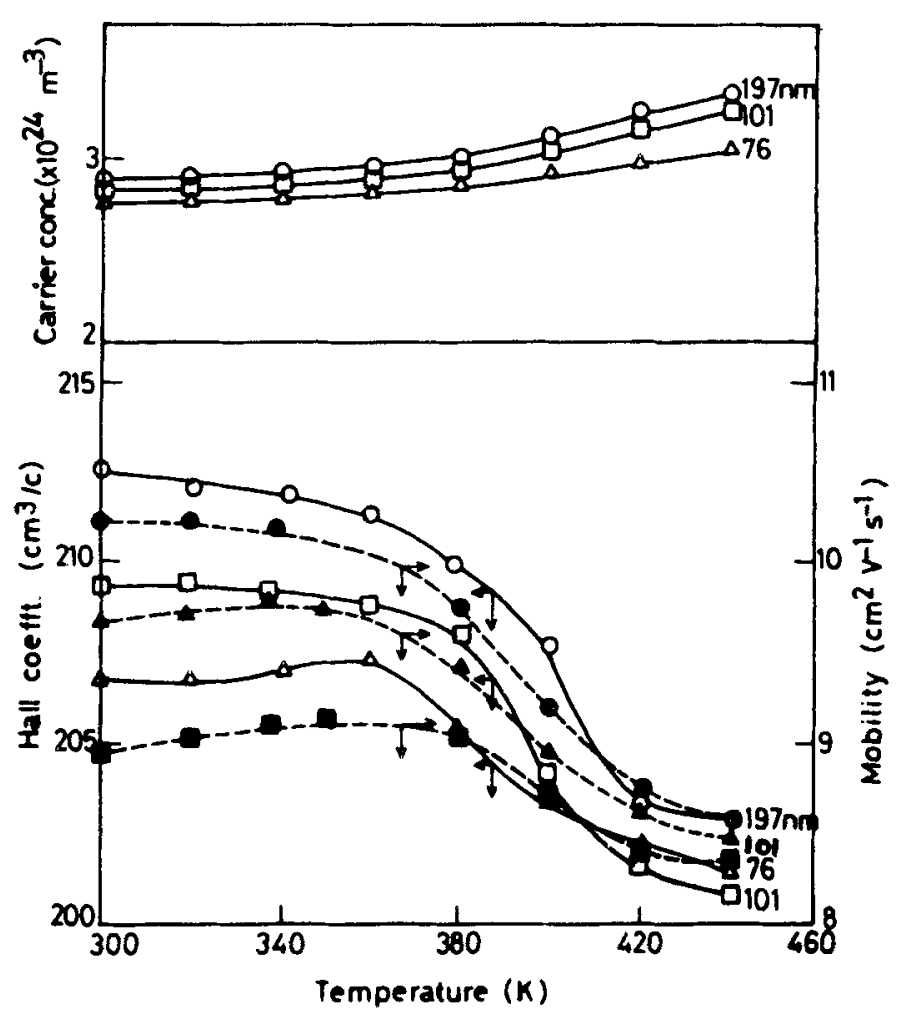

Figure 15. a. Variation of Hall coefficient with different temperatures, b. variation of Hall mobility with temperatures and c. plot of carrier concentration with temperatures.

The carrier concentrations for different film thicknesses are plotted in figure $15 \mathrm{c}$. Carrier concentration increases only weakly with temperature and we could not find a significant dependence on thickness.

All the samples appear to be $p$ type which indicates that the current transport is primarily due to holes as a result of thermal excitation of electrons and their subsequent capture by acceptor states. The thermoelectric power was estimated to be $210 \mu \mathrm{V} \mathrm{K}^{-1}$

\section{Conclusions}

Tellurium thin films formed by vacuum evaporation method possess polycrystalline structure. Annealing of the films causes reduction in defects. In the low frequencies and at higher temperatures the capacitance is dependent both on temperature and frequency whereas it is independent of frequency at low temperatures and high frequencies. The observed low activation energy in a.c. conduction mechanism suggests that the conduction in these films may be due to electronic hopping. Also current-voltage characteristics for different field and temperatures were studied and it has been found that the conduction process is space charge limited (SCLC). The activation energy has been found to be associated with the traps in the energy gap and is field dependent. The free-carrier mobility, concentration of charge carriers, effective density of states in the conduction band and density of trapped electrons and height of the Schottky barrier in 
the process have also been calculated. The Hall coefficient and carrier mobility are also discussed. It is concluded that the thermally evaporated tellurium films were $p$ type.

\section{References}

Balasubramaniam T, Narayandass Sa K, Balasubramanian C and Mangalaraj D 1991 Proc. Solid State phys. Symp. India C34245

Balasubramaniam T, Narayandass Sa K and Mangalaraj D 1995 National conference in thin film processing and applications (Tirupati: SV Univ.) p. 33

Berry W B and Jayadereiah T S 1969 Thin Solid Films 377

Birey H 1978 J. Appl. Phys. 492898

Capers M J and White M 1971 Thin Solid Films 8317

Capers M J and White M 1973 Thin Solid Films 155

Chaudhuri A K and Duttaray S K 1965 Indian J. Phys. 39156

Chaudhuri A K and Bose H N 1977 Indian J. Phys. 49783

Dinno M A, Sehwartz M and Giammare B 1974 J. Appl. Phys. 453328

Easwaran N, Balasubramanian C, Narayandass Sa K and Mangalaraj D 1992 Phys. Status Solidi (a)129 443

Goswami A and Goswami A P 1977 Pramana-J. Phys. 8335

Goswami A and Ojha M 1973 Thin Solid Films 16187

Gould R D 1982 J. Appl. Phys. 533353

Gould R D and Hogarth C A 1975 Int. J. Electron. 38577

Gutierrez W A, Feldman C A and Layton W T 1962 Bull. Am. Phys. Soc. 758

Harrop P J 1972 Dielectrics (London: Butterworth)

Hill R M 1971 Philos. Mag. 2359

Hughes D M and Jones M W 1974 J. Phys. D 72081

Jonscher A K 1973 J. Phys. C Solid Phys. 61235

Kannan M D, Narayandass Sa K, Balasubramanian C and Mangalaraj D 1990 Phys. Status Solidi A121 515

Kannan M D, Narayandass Sa K, Balasubramanian C and Mangalaraj D 1992 J. Mater. Sci. 275040

Kobory A and Janda M 1977 Phys. Status Solidi A40 225

Lakshminarayan N, Radhakrishnan M and Balasubramanian C 1982 J. Mater. Sci. 171623

Lancaster M C 1972 J. Phys. D 51133

Leistiko O, Grove A S and Shah T C 1965 IEEE Trans. Electron. Dev. 2248

Mahalingam T, Radhakrishnan M and Balasubramanian C 1981 Thin Solid Films 78229

Nakane H, Noya A, Kuriki S and Matsumoto 1979 Thin Solid Films 59291

Nikam P S and Aher H S 1993 Indian J. Pure and Appl. Phys. 3179

Nikam P S and Aher H S 1994 Bull. Mater. Sci. 1741

Okuyama K, Yamashita T, Ehiba M and Kumagai Y 1977 Jpn. J. Appl. Phys. 161571

Orlowski T, Struzik M and Szaro L 1977 Thin Solid Films 40 L1-L3

Peek D, Guerra V C and Sladek R J 1985 J. Appl. Phys. 574803

Phahle A M 1977 Thin Solid Films 41235

Rapos M, Ruzinsky M, Luby S and Cervenk J 1976 Thin Solid Films 36103

Reucroft P J and Ghosh S K 1974 Thin Solid Films 20363

Roberts G G, Keeting B S and Shelly A V 1974 J. Phys. D (GB) 71595

Sasi N, Balasubramanian C and Narayandass Sa K 1987 Phys. Status Solidi (a)103 475

Soukup R J and Speliotis D E 1970 J. Appl. Phys. 413229

Subbrayan A, Balasubramanian C and Narayandass Sa K 1988 Indian J. Pure and Appl. Phys. 26410

Tolansky S 1948 Multiple beam interferometry of surfaces and films (London: Oxford University Press) p. 147

Wieder H H 1965 Proc. int. symp. on basic problems in thin film physics (Gottingen: Clausthal) p. 719 